\title{
Report
}

\section{Comparison of topologies suitable for capacitor charging systems}

\author{
J-M. Cravero ${ }^{1}$, S. Maestri ${ }^{2}$, R. Garcia Retegui ${ }^{2}$, M. Benedetti ${ }^{2}$, G Uicich $^{2}$ \\ 1 CERN \\ 2 Universidad de Mar del Plata, Argentina
}

Keywords: Pulsed power converter, high voltage power converters, particle accelerator

\begin{abstract}
This paper presents a comparison between topologies suitable for capacitor charging systems.

The topologies under evaluation are a yback converter, a half-bridge series resonant converter and a full-bridge phase-shifted converter. The main features of these topologies are highlighted, which allows the proper topology selection according to the application requirements. Moreover, the performed analysis permits to characterize the operational range of the main components thus allowing their appropriate sizing and selection. Simulation results are provided.
\end{abstract}

Presented at:

EPE

Lille, France

September, 2013 


\title{
Comparison of topologies suitable for Capacitor Charging Systems
}

\author{
${ }^{\dagger}$ Sebastián Maestri, ${ }^{\dagger}$ Rogelio Garcia Retegui, ${ }^{\dagger}$ Gustavo Uicich, \\ ${ }^{\dagger}$ Mario Benedetti ${ }^{\ddagger} J$ Jean Marc Cravero. \\ $\dagger$ UNIVERSIDAD NACIONAL \\ $\ddagger$ EUROPEAN ORGANIZATION FOR \\ DE MAR DEL PLATA \\ L.I.C. \\ Juan B.Justo 4302 \\ Mar del Plata, Argentina \\ Phone: +54 2234816600 \\ Fax: +54 2234810046 \\ NUCLEAR RESEARCH - C.E.R.N. \\ TE-EPC GROUP \\ 1211 Geneva 23 \\ Geneva, Switzerland \\ Phone: +412276722 23 \\ Fax: +41227665300 \\ Email: somaestri@fi.mdp.edu.ar \\ URL: http://www3.fi.mdp.edu.ar \\ Email:Jean-Marc.Cravero@cern.ch \\ URL: http://www.cern.ch
}

\section{Acknowledgments}

This work was supported in part by the National University of Mar del Plata (UNMDP), Argentina, the National Scientific and Technical Research Council (CONICET), Argentina, by the Ministry of Science, Technology and Productive Innovation (MINCYT), Argentina, by the National Agency of Scientific and Technological Promotion, Argentina, by the European Organization for Nuclear Research (CERN), Switzerland, and by the European Particle Physics Latin American Network (EPLANET).

\section{Keywords}

$<<$ Pulsed power converter $>>,<<$ High voltage power converters $>>,<<$ Particle accelerator $>>$.

\begin{abstract}
This paper presents a comparison between topologies suitable for capacitor charging systems. The topologies under evaluation are a flyback converter, a half-bridge series resonant converter and a full-bridge phase-shifted converter. The main features of these topologies are highlighted, which allows the proper topology selection according to the application requirements. Moreover, the performed analysis permits to characterize the operational range of the main components thus allowing their appropriate sizing and selection. Simulation results are provided.
\end{abstract}

\section{Introduction}

In particle accelerators, several applications are based on a capacitor discharge topology $[1,2]$. In this kind of systems, the capacitor charging system plays an essential role, since it has to transfer from the grid to the capacitive bank the energy that has to be further delivered to the load. The capacitor charging system has to exhibit several features, among others pulse-to-pulse repeatibility, compactness, efficiency and robustness $[2,3]$. There are many topologies suitable for capacitor charging systems, each one having advantages and disadvantages $[4,5,6,7]$. This paper presents the comparison between three topologies, a dual switch flyback working in the boundary between CCM (Continuous Conduction Mode) and DCM (Discontinuous Conduction Mode), a full-bridge phase-shifted converter and a half-bridge series resonant converter. Several features of each topology are highlighted and a comparison is performed. The presented analysis evaluates the capacitor charger features that are useful to select the more suitable topology, 
depending on the requirements of a particular application. Moreover, the presented expressions allow to define the operational range of the different components thus allowing their appropriate sizing and selection according to clearly defined parameters.

\section{Capacitor Charging Systems}

There are several parameters that influences the charging system [3]. Among them, the Pulseto-Pulse Repeatability $(P P R)$ is one of the most important since it defines the variation in the final charging voltage. $P P R$ can be defined as:

$$
P P R=\frac{\left(v_{0 \max }-v_{0 \min }\right)}{v_{0 \text { avg }}} \cdot 100 \%
$$

where $v_{0 \text { max }}, v_{0 \text { min }}$ and $v_{0 \text { avg }}$ are the maximum, minimum, and average values of the voltage across the output capacitor $C_{o}$ for a batch of pulses. Usually, the charging process is finished when the output voltage reaches its reference value, $V_{R}$. With many charging topologies, the output voltage exhibits an overshoot due to the inherent remanent energy stored in the power converter elements, $E_{r e m}$. $P P R$ can also be defined as a function of this remanent energy and the maximum energy in the capacitor, $E_{c}$ :

$$
P P R=\left(\sqrt{1+\frac{E_{r e m}}{E_{c}}}-1\right) \cdot 100 \%
$$

where $E_{c}=1 / 2 \cdot C_{o} \cdot V_{M A X}{ }^{2}$ and $V_{M A X}$ is the maximum charging voltage. A similar parameter that defines the quality of the charging system is the voltage error between the end of charge voltage and the reference voltage, $\Delta V_{o}=V_{E O C}-V_{R}$, relative to the target voltage, see Eq. (3). Notice that $P P R=\frac{\Delta V_{o}}{V_{R}}$ for $V_{R}=V_{M A X}$.

$$
\frac{\Delta V_{o}}{V_{R}}=\frac{V_{E O C}-V_{R}}{V_{R}}=\frac{V_{E O C}}{V_{R}}-1
$$

Other figures of merit of charging systems are related to the power capability[4]. The most relevant are its output charging power and average charging power. The average power is tied to the application requirements and it relates the repetition rate, the target output voltage and the capacitor value, as shown in Eq. (4):

$$
P_{a v}=\frac{C_{o} V_{R}^{2}}{2 \cdot T_{P}} \quad[\mathrm{~W}]
$$

The charging power determines some parameters of the power converter associated to the charging process and it relates the time necessary to reach the target output voltage and the capacitor value, as can be seen in Eq. (5):

$$
P_{C H}=\frac{C_{o} V_{R}^{2}}{2 \cdot t_{C H}} \quad[\mathrm{~J} \cdot \mathrm{s}]
$$

These features will define some specifications on the power converter used for the capacitor charging system, among them the average output current, the maximum current on the devices and its switching frequency. These specifications will be analyzed in the following sections for the selected topologies. In order to define a framework for the comparison, the three topologies are evaluated with the same charging time and capacitive load so the same charging power.

\section{Evaluated topologies}

\section{Dual switch flyback converter}

Flyback converters have been used for capacitor charging systems [6]. Figure 1 shows a simplified scheme of a dual switch flyback converter. This topology is based on the use of two transistors at the primary side, which are turned on and off simultaneously. The converter is operated in the boundary between CCM and DCM, this operation mode reduces the transient power losses, minimizes the transformer size and avoids a primary overcurrent during the initial stage of capacitor charging. Besides, it provides a fast transient response. 


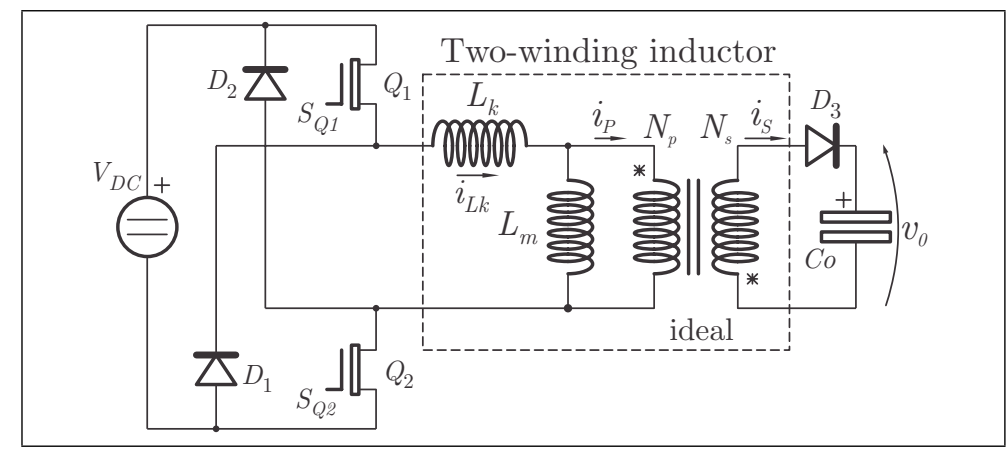

Figure 1: Dual switch flyback converter.

The operation principle of this control mode can be summarized as follows:

- The first switching cycle is initiated turning on $Q_{1}$ and $Q_{2}$. The input voltage, $V_{D C}$, is applied on the primary side inductance, $L_{m}$. The secondary voltage reverse-biases the $D_{3}$ diode and no energy is transferred to the output capacitance. Thus, the primary current increases linearly and the energy is stored in the core of the two-winding inductor.

- When the primary current reaches the peak current reference, $\hat{i_{p}}, Q_{1}$ and $Q_{2}$ are turned off. The energy stored in the two-winding inductor core forward-biases the $D_{3}$ diode and current flows into the output capacitor. The secondary side can be analyzed as a resonant circuit $L_{s} C_{o}$, with $L_{s}=N^{2} L_{m}$ and $N=N_{s} / N_{p}$. Concerning the energy stored in the primary-winding leakage inductance, it flows through $D_{1}$ and $D_{2}$ and returns to the input source. Therefore, the maximum voltage applied on the primary side semiconductor devices is clamped to the input voltage. Besides, in case of load disconnection, the energy stored in the two-winding inductor returns through the diodes to the input source.

- A new switching cycle is initiated when the secondary current reaches zero.

An aspect that is non-optimal in this topology is that the turn-off power losses are significantly greater than the turn-on power losses. A snubber network should be added for turn-off, which will allow obtaining a ZCS (Zero Current Switching) and ZVS (Zero Voltage Switching) converter. An important feature is given by the fact that the inductor peak current is constant in the first half of the switching cycle. On the other hand, the output capacitor voltage is variable along the charging cycle and consequently the off time is variable with the output voltage. Hence, the commutation frequency in each $j$ interval is variable and depends on the output voltage, leading to a voltage precision that depends on the operating point. The main equations of this converter are:

$$
\begin{aligned}
\hat{i_{Q_{m a x}}} & =\frac{V_{D C}}{L_{m}} t_{o n} \\
\left\langle i_{C_{o}(j)}\right\rangle & =\frac{f_{s w(j)}}{\omega_{o}} \sqrt{\left(\frac{\hat{i_{p}}}{N}\right)^{2}+\left(\frac{v_{0(j-1)}}{Z_{o}}\right)^{2}}\left(1+\cos \phi_{j}\right) \\
f_{s w(j)} & =\frac{1}{\frac{L_{m} \hat{i_{p}}}{V_{D C}}+\frac{\phi_{j}}{\omega_{0}}} \\
\frac{\Delta V_{0}}{V_{R}} & =\sqrt{1+\frac{L_{m}}{C_{o}}\left(\frac{\hat{i_{p}}}{V_{R}}\right)^{2}}-1
\end{aligned}
$$

where $v_{0}$ is the output voltage, $j$ is the time interval between the rising edges of the ON signal, $\phi_{j}=\arctan \left(\frac{\hat{i_{p}} Z_{0}}{N v_{0}(j-1)}\right), Z_{0}=\sqrt{L_{s} / C_{o}}$ and $\omega_{0}=1 / \sqrt{L_{s} C_{o}}$.

\section{Full-bridge phase-shifted converter}

Full-bridge topologies are widely used in high-power, high-voltage conversion $[4,7]$. Figure 2 shows a full-bridge phase-shifted converter. 


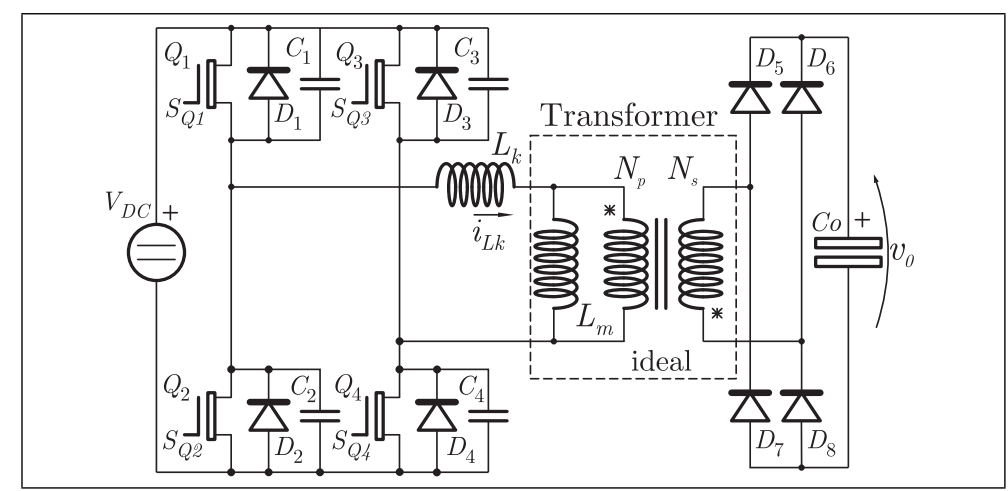

Figure 2: Full-bridge phase-shifted converter.

The system controls each leg in a complementary and independent way, using the time delay $\left(t_{d}\right)$ between the turn-on of the pair $Q_{1}-Q_{4}$ and the turn-on of the pair $Q_{2}-Q_{3}$ to regulate the load voltage. The voltage applied at the primary side of the transformer is rectified using diodes $D_{5-8}$ and the converter maximum output voltage can be sized by tuning the $T_{1}$ transformer ratio $N=N_{s} / N_{p}$. In this topology, the voltage at the primary side of the transformer between pulses can be considered as a voltage source, being $v_{0}^{\prime}=\frac{N_{p}}{N_{s}} v_{0}$.

Full-bridge topologies are usually used in order to achieve ZVS at the turn-on of primary side switches. In this case, the power losses are greatly reduced, due to the lossless capacitors used as snubbers that act during turning-off $[7,8,9,10]$. If the connection to the isolating transformer is realized with a LC leg, a resonant behaviour is obtained, leading to the operation with quasi-sinusoidal waveforms. This kind of topology is named Full-Bridge Phase-Shifted Series loaded Resonant Converter (FBPS-SRC). Although it offers the benefits of resonant converters as low switching losses, the design in this kind of topologies becomes complex since the different operating points have to be numerically solved [10]. Moreover, the relationship between the switching frequency and the resonance frequency is a trade-off between maximizing the energy delivered to the load and avoiding an oscillating behaviour. Another option, which is the one analyzed in this article, is to use a single inductor. This topology, named Full-bridge Phase-Shifted Zero Voltage Transition Converter (FBPS-ZVTC), leads to linear currents, which simplifies the analysis and allows the realisation of an analytical design.

The main advantages of this kind of converter are a fixed switching frequency in each $j$ interval, a relatively simple design and the possibility of ZVS-ZCS on the primary side switches. Moreover, lossless snubbers allows decreasing switches power losses without sacrificing efficiency. A disadvantage that has to noted is that ZVS-ZCS conditions is achieved in a specific operation mode, i.e. ZVS-ZCS is strongly dependent on the load. There are topological modifications that assure the desired operation mode. However, the complexity of the system is greatly increased [10]. Furthermore, since the inductor current decay depends on the output voltage, the inductor current at the beginning of the charge and in case of output load short-circuit is unipolar. Consequently, a strategy to avoid core saturation must be implemented. The main equations of this converter are:

$$
\begin{aligned}
\hat{i_{Q_{\max }}} & =\hat{i_{D \max }}=\frac{V_{D C}-v_{0(j)}^{\prime}}{2 L_{k}}\left(t_{o n}-\frac{t_{d}}{2}\right) \\
\left\langle i_{C_{o}(j)}\right\rangle & =\frac{V_{D C} / 2}{N T L_{k}}\left[t_{o n}^{2}-\left[t_{d}-\frac{v_{0(j)}^{\prime} t_{o n}}{V_{D C}}\right]^{2}-\frac{2 V_{o(j)}^{\prime}}{V_{D C}}\left[t_{d}^{2}+t_{d} t_{o n}\right]\right] \\
f_{s w} & =\frac{1}{T}=\frac{d}{t_{o n}} \\
\frac{\Delta V_{0}}{V_{R}} & \approx \sqrt{1+\frac{C_{n}}{C_{o}}\left(\frac{V_{D C}}{V_{R}}\right)^{2}}-1
\end{aligned}
$$

where $f_{s w}$ is the switching frequency and $C_{n}$ is the value of the snubber capacitors. 


\section{Half-bridge series resonant converter}

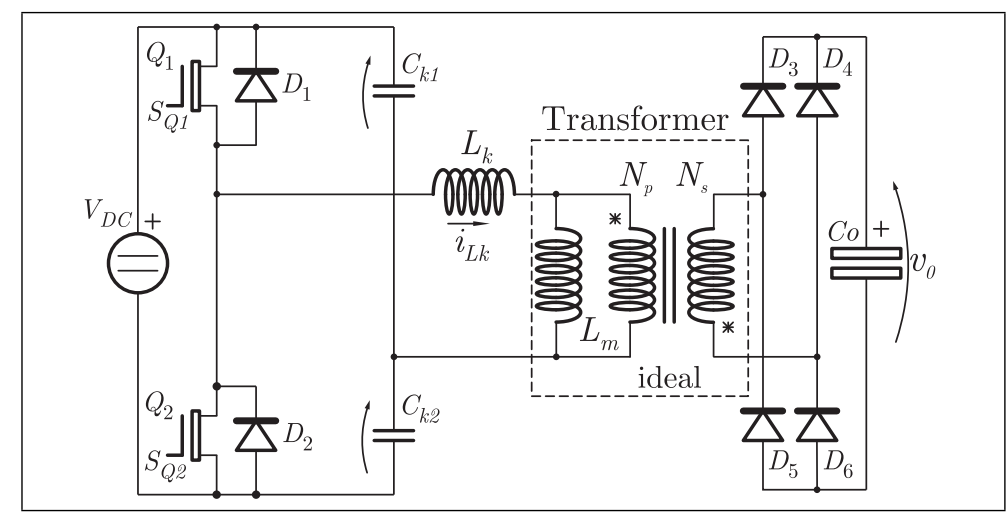

Figure 3: Half-bridge series resonant converter.

Figure 3 shows a half-bridge series resonant converter. This topology is widely used in capacitor charging systems due to its simplicity and robustness [5]. By turning-on $Q_{1}$ or $Q_{2}$, a resonant circuit between $L_{k}, C_{k 1}, C_{k 2}, V_{D C}$ and the load, which is reflected to the primary side through $N_{p} / N_{s}$, is formed. Inductor $L_{k}$ represents the inductances in series with the transformer, including its own leakage inductance. In general, the value of $C_{o}$ reflected to the primary side is much greater than $C_{k 1}$ and $C_{k 2}$. Therefore, $C_{o}$ does not modify the resonance frequency and, between pulses, it can be considered as a voltage source $v_{0}^{\prime}=\frac{N_{p}}{N_{s}} v_{0}$. Hence, the resonant frequency is given by:

$$
\begin{aligned}
& \omega_{k}=2 \pi f_{k}=\sqrt{\frac{1}{L_{k}}\left(\frac{1}{C_{k}}+\frac{1}{C_{o}^{\prime}}\right)} \approx \frac{1}{\sqrt{L_{k} C_{k}}} \\
& Z_{k}=\sqrt{\frac{L_{k}}{C_{k}}}
\end{aligned}
$$

where $C_{k}=C_{k 1}+C_{k 2}$ and $C_{o}{ }^{\prime}=\left(\frac{N_{s}}{N_{p}}\right)^{2} C_{o}$. In this topology, $T_{1}$ acts as an isolating transformer, i.e. there is no energy storage. Then, the secondary side voltage is rectified using diodes $D_{3-6}$ and delivered to the capacitor. In order to obtain a constant current mode operation, there is a dead time, $T_{d}$, between the turning-on of $Q_{1}$ and $Q_{2}$. Hence, the system works in DCM.

The operation principle of this topology can be summarized as follows:

- The first switching cycle is initiated by turning on $Q_{1}$. A resonant circuit given by $V_{D C}$, $C_{k 1}, C_{k 2}$ and $L_{k}$ is formed, leading to sinusoidal waveforms for the inductance current and capacitor voltages. The transformer reflects on the primary side the output capacitor. Due to its large value, this capacitor does not affect the resonance and it can be considered as a constant voltage source.

- When the inductor current becomes negative, $Q_{1}$ stops conducting and $D_{1}$ becomes forward biased, carrying the inductor current. At this time, the control signal of $Q_{1}$ can be set to OFF. The first cycle ends when $D_{1}$ stops conducting.

- After a dead time $T_{d}, Q_{2}$ is turned-on and the cycle is repeated with $Q_{2}$ carrying the inductor current.

Capacitor chargers based on this topology acts as a current source, which allows reducing the inrush current. Furthermore, the output voltage grows linearly and the output power is a linear function of time [3]. The main equations of this converter are: 


$$
\begin{aligned}
\hat{i_{Q_{\max }}} & =\frac{V_{D C}}{2 Z_{k}}\left(1+2 \frac{V_{R} \cdot N_{p} / N_{s}}{V_{D C}}\right) \quad \hat{i_{D \max }}=\frac{V_{D C}}{2 Z_{k}}\left(1-2 \frac{V_{R} \cdot N_{p} / N_{s}}{V_{D C}}\right) \\
\left\langle i_{C_{o}}\right\rangle & =\frac{2 f_{s w}}{f_{k}} \frac{V_{D C}}{\pi Z_{k}} \frac{N_{p}}{N_{s}} \\
f_{s w} & =\frac{1}{2\left(\frac{1}{f_{k}}+T_{d}\right)} \\
\frac{\Delta V_{0}}{V_{R}} & =\frac{V_{D C}}{N \cdot V_{R}} \frac{C_{k}}{C_{o}}\left(1-\frac{1}{N} \frac{V_{R}}{V_{D C}}\right)
\end{aligned}
$$

\section{Comparison}

In order to perform a comparison, the capacitor charger systems were designed to obtain the same charging time, i.e. the same charging power. Therefore, the output current averaged on a time $t_{c h}$ is the same for the three topologies. The most relevant features of the system are: $V_{M A X}=1000 \mathrm{~V}, C_{o}=2.7 \mathrm{mF}, t_{c h}=1.2 \mathrm{~s}, T_{P}=1.35 \mathrm{~s}$ and $V_{D C}=400 \mathrm{~V}$. According to Eq. (4), $P_{a v}=1 \mathrm{~kW}$. Concerning the evaluated topologies, their main parameters are summarized as follows:

Table I: Main parameters of the evaluated topologies.

\begin{tabular}{ccc}
\hline DSFC & FBPSC & HBSRC \\
\hline$L_{m}=300 \mu \mathrm{H}$ & $L_{k}=30 \mu \mathrm{H}$ & $L_{k}=7 \mu \mathrm{H}$ \\
$N=3$ & $N=6.5$ & $N=5.5$ \\
$\hat{i_{P}}=19 \mathrm{~A}$ & $C_{n}=2 \mathrm{nF}$ & $C_{k}=76 \mathrm{nF}$ \\
& $f_{s w}=100 \mathrm{kHz}$ & $f_{s w}=100 \mathrm{kHz}$ \\
& $t_{d}=1.25 \mu \mathrm{s}$ & \\
\hline
\end{tabular}

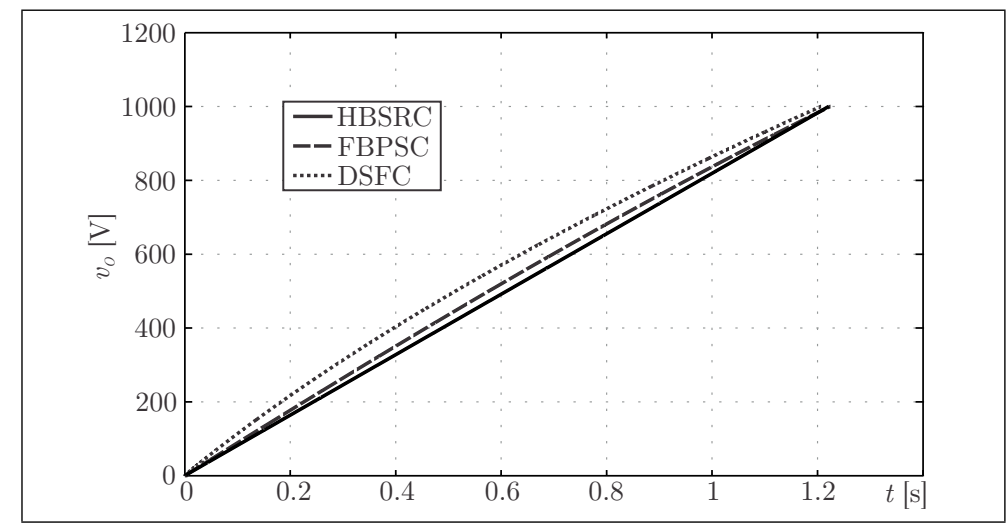

Figure 4: Output voltage.

Figure 4 shows the output voltage for the implemented systems. It can be noted that the charging time is approximately the same for the three chargers. The $H B S R C$ performs a linear charging process, while with the other topologies the charging voltage slope is variable. The slope variation is most significant for the $D S F C$. Moreover, for target voltages lower than $V_{M A X}$, the $D S F C$ has the maximum voltage slope, which implies that this topology has the higher charging power.

Figure 5 depicts the output current, averaged on a switching interval, as a function of the output voltage. This figure shows that the $H B S R C$ produces a constant average current, while the other ones present a variable average current. It should be noted that the average current behaviour is related to the linearity of the charging process. Moreover, DSFC and FBPSC have maximum average current at the beginning of the charge and then it decreases when the output voltage increases. This feature implies that these topologies have a charging power higher than the $H B S R C$ for low voltages, as shown in Fig. 4. 


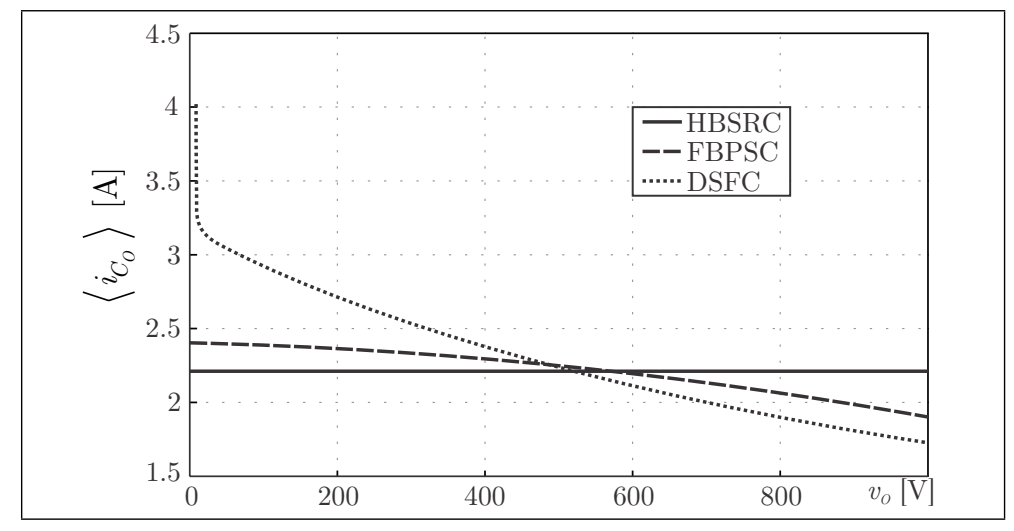

Figure 5: Average output current.

Figure 6 shows the output voltage precision, i.e. $\Delta V_{o} / V_{R}$, for different cases of target voltages. It can be noted that in the three topologies the precision is increased when the output voltage is higher. For the presented designs, the HBSRC presents better precision when the charging voltage is low, while for higher voltages the $F B P S C$ presents a superior precision. This parameter is strongly dependent on the design adopted and it can be adjusted (considering the same average output current) by using the design equations Eq. (6), Eq. (7) and Eq. (9).

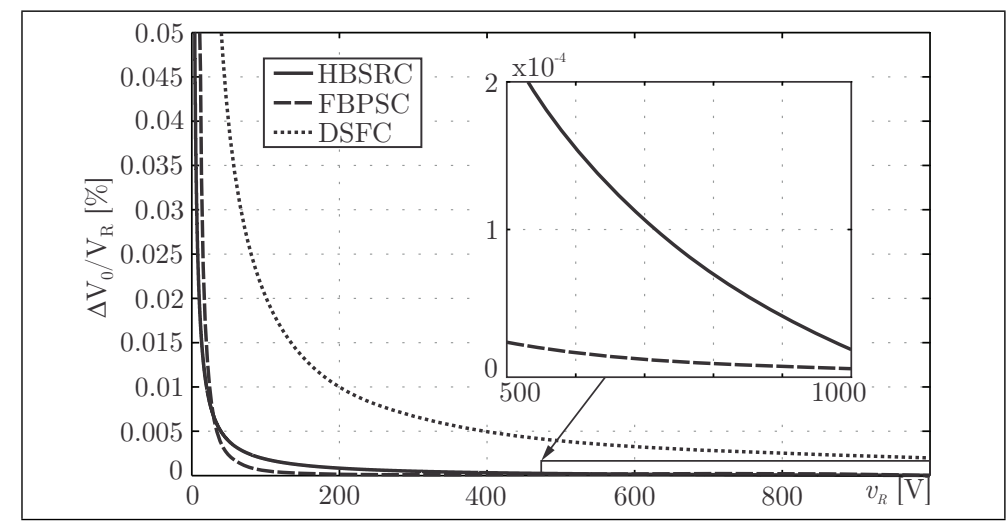

Figure 6: Output voltage precision.

\section{Simulations}

In order to evaluate the topologies, the capacitor charging systems, using previously mentioned parameters, were simulated with MATLAB SIMULINK. The simulation figures show the main capacitor charger waveforms, i.e. the charging current, $i_{S}$, and the output voltage, $v_{0}$.

Figure 7 shows the waveforms of $D S F C$ topology. Figure 7 (a) depicts a detail of the beginning of the charging process, where the variable frequency operation of the system can be noted. Furthermore, it can be seen that the output voltage step decreases as $v_{0}$ increases, which is related in this topology to the low precision at low charging voltages. The end of charge is shown in Fig. 7(b), where it can be seen that the variation of switching frequency is lower than in the beginning of charge situation. Moreover, the voltage precision is improved. Concerning $i_{S}$, it has a high $d i / d t$ at the beginning of each pulse, which implies demanding operating conditions for the output diode.

Figure 8 shows the waveforms of $F B P S C$ topology. It can be seen that in both the beginning and the end of charging process the voltage steps are different, due to the small variation in the output current pulses. Moreover, it can be noted that when $i_{S}$ reaches its maximum, the falling slope of the current during $t_{d}$ is higher as $v_{0}$ increases. In this case, the waveform on the output diodes is less demanding than in the $D S F C$ topology. 


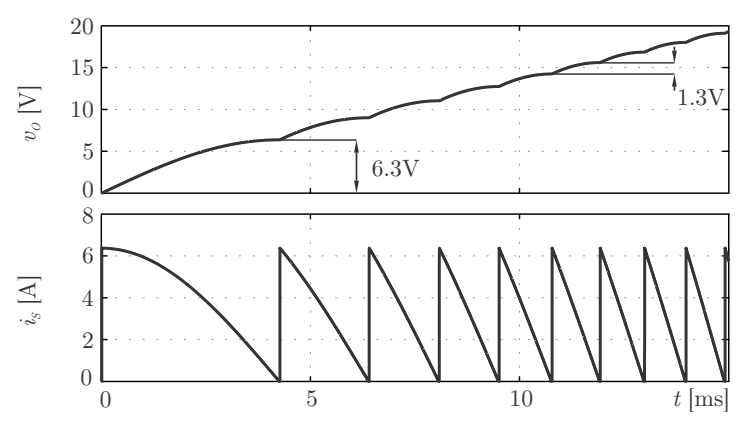

(a)

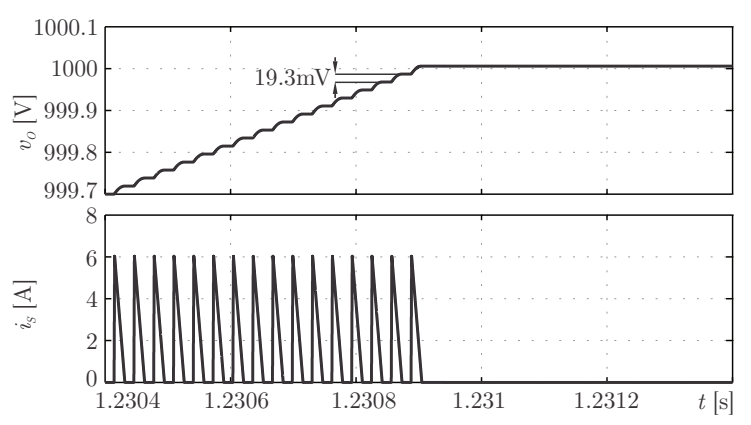

(b)

Figure 7: Double-switch flyback converter. Output voltage (upper) and output current (lower). Beginning of charging process (a) and End of of charging process (b).

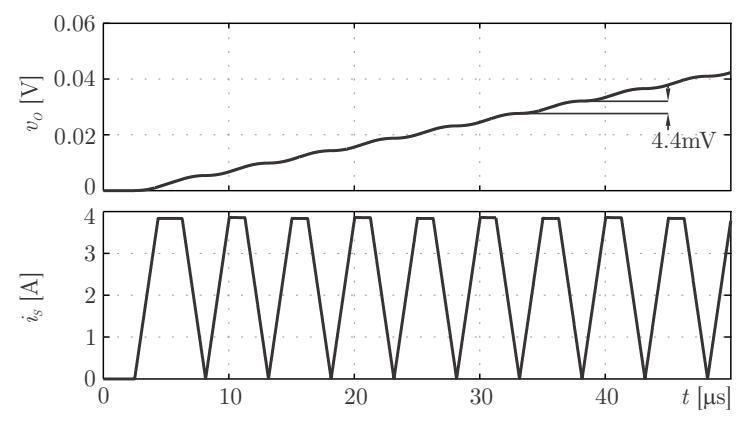

(a)

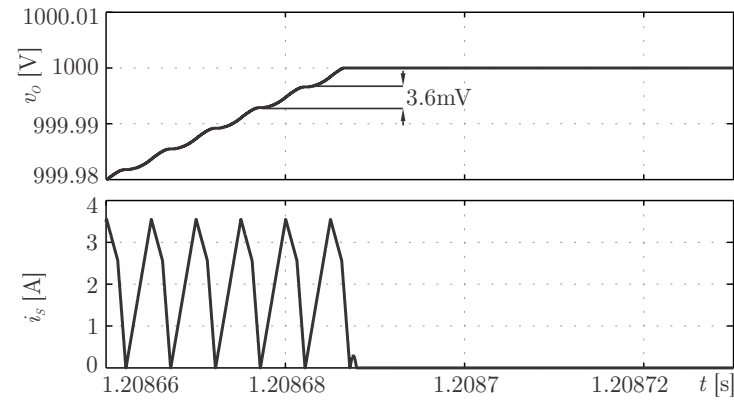

(b)

Figure 8: Full-bridge phase-shifted converter. Output voltage (upper) and output current (lower). Beginning of charging process (a) and End of of charging process (b).

Figure 9 shows the $H B S R C$ waveforms. In this case, the output voltage step is constant during the whole range of target voltage. As stated in Eq. (9), when the output voltage reflected to the primary side of the transformer is low compared with $V_{D C}$, the maximum values of current for the transistors and the primary side diodes are similar. When the output voltage increases, the peak current of transistors are higher than the ones of the diodes. This situation can be noted in Fig. 9(b), where the rectified waveform presents two different maximum amplitudes. Despite of this variation, the average output current remains constant in each switching interval, as stated in Fig. 5. Finally, it should noted that the current in the output diodes is sinusoidal, which implies lower constraints on the diodes sizing.
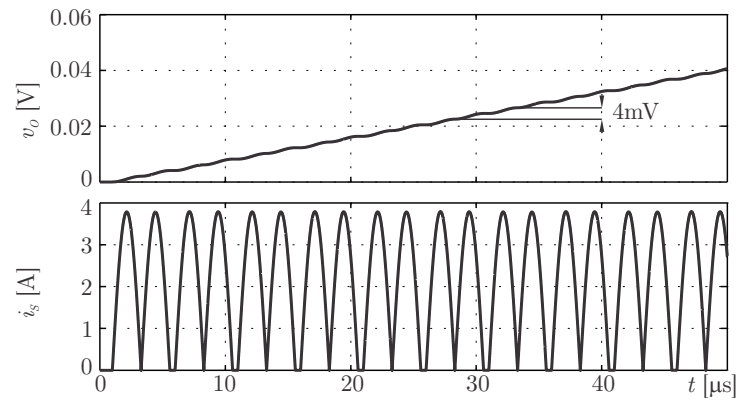

(a)

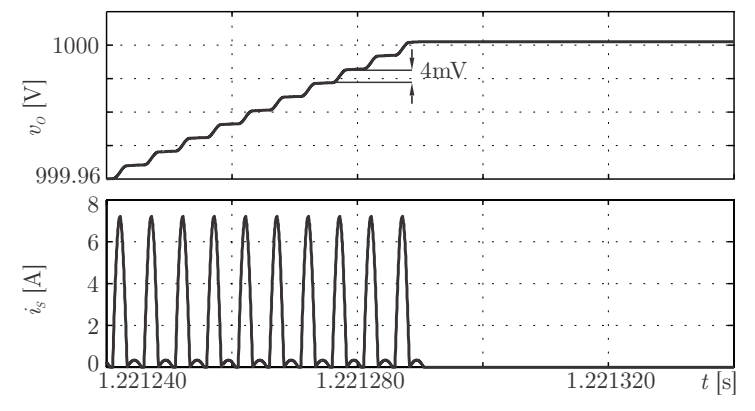

(b)

Figure 9: Half-bridge series resonant converter. Output voltage (upper) and output current (lower). Beginning of charging process (a) and End of the charging process (b). 


\section{Conclusions}

A comparison of topologies suitable for capacitor charging systems has been presented. The most significant aspects of this kind of systems have been considered. Three topologies have been evaluated: a dual switch DCM flyback, a full-bridge phase-shifted converter and a halfbridge series resonant converter. Simulations have been carried out to point out the performances and important features of each topology thus allowing the determination of the optimal topology as regard to the application requirements and component sizing.

\section{References}

[1] M. Souda, F. Endo, and C. Yamazaki, "Development of high power, high voltage dc power supply for pulsed-power applications and accelerators," in High-Power Particle Beams, 2000 13th International Conference on, june 2000, pp. $930-933$.

[2] J. Oh, S. Jang, Y. Son, M. Cho, and W. Namkung, "Development of a capacitor-charging power supply for a smart modulator," in Proceedings of the Second Asian Particle Accelerator Conference, Beijing, China, 2001, june 2001, pp. $719-721$.

[3] A. Pokryvailo, C. Carp, and C. Scapellati, "High-power high-performance low-cost capacitor charger concept and implementation," Plasma Science, IEEE Transactions on, vol. 38, no. 10, pp. 2734 2745 , oct. 2010.

[4] A. Lippincott and R. Nelms, "A capacitor-charging power supply using a series-resonant topology, constant on-time/variable frequency control, and zero-current switching," Industrial Electronics, IEEE Transactions on, vol. 38, no. 6, pp. $438-447$, dec 1991.

[5] M. Wolf and A. Pokryvailo, "High voltage resonant modular capacitor charger systems with energy dosage," in Pulsed Power Conference, 2005 IEEE, june 2005, pp. 1029 -1032.

[6] J. Elmes, C. Jourdan, O. Abdel-Rahman, and I. Batarseh, "High-voltage, high-power-density dcdc converter for capacitor charging applications," in Applied Power Electronics Conference and Exposition, 2009. APEC 2009. Twenty-Fourth Annual IEEE, feb. 2009, pp. 433 -439.

[7] S. Jang, S. Ahn, H. Ryoo, and G. Rim, "Novel high voltage capacitor charger for pulsed power modulator," in Power Modulator and High Voltage Conference (IPMHVC), 2010 IEEE International, may 2010 , pp. $317-321$.

[8] S. Lakshmikanth, T. Girikumar, and A. U. Rani, "Design of phase shift resonant converter using zero voltage switching," in Information and Communication Technology in Electrical Sciences (ICTES 2007), 200\%. ICTES. IET-UK International Conference on, dec. 2007, pp. $210-214$.

[9] Y. Kelkar, Y. Raikwar, and A. Thakurta, "A capacitor charging power supply using series resonant topology," in Indian Particle Accelerator Conference - 2009 (InPAC-09) , RRCAT, Indore, february 2009 , pp. $1-3$.

[10] B. Nathan and V. Ramanarayanan, "Analysis, simulation and design of series resonant converter for high voltage applications," in Industrial Technology 2000. Proceedings of IEEE International Conference on, vol. 1, jan. 2000, pp. $688-693$ vol.2. 